Int. J. Speleol. 5 (1973), pp. 325-336.

\title{
II popolamento di ragni nelle grotte tropicali (Araneae)
}

\author{
di
}

\author{
Paolo Marcello BRIGNOLI*
}

Gli Aracnidi, nel loro insieme, sono un gruppo ampiamente rappresentato nell'ambiente cavernicolo; anzi, dopo la recente scoperta di Scorpioni forse troglobi (cavernicoli lato sensu in ogni caso), tutti gli ordini, con la sola eccezione dei Solifugi, gruppo spiccatamente xerofilo, sono più o meno frequenti nelle grotte.

Tra tutti, i più comuni, assenti solo eccezionalmente, sono i ragni. Rispetto al numero totale di specie note (circa 30.000), quelle cavernicole, tra troglofili e troglobi, sono poche centinaia ; varie di queste però sono tra gli animali cavernicoli più abbondanti.

I ragni sono tutti predatori; dalla loro frequenza e, spesso, abbondanza numerica nelle grotte, si può senz'altro desumere che in questo ambiente essi sono probabilmente tra $i$ predatori più importanti e, forse, in molti casi, $i$ principali in senso assoluto.

I modi utilizzati dai ragni per catturare le prede sono essenzialmente tre: mediante la tela, di estensione, struttura e robustezza variabile; l'agguato, stando immobili su fiori o in tane nel terreno; la ricerca attiva, di solito a vista. Si potrebbe pensare che gli ultimi due tipi di caccia diano scarsi risultati nell'ambiente cavernicolo e ciò, almeno per le grotte dei paesi temperati, dalla fauna piuttosto rarefatta, corrisponde a realtà; in questo tipo di grotte infatti vi sono pochissimi predatori vaganti (per lo più Dysderidae ciechi, utilizzanti il tatto), mentre non vi è praticamente nessuna specie che stia all'agguato.

Quasi tutti i ragni delle grotte temperate costruiscono tele, ad altezza variabile dal suolo. Nelle grotte tropicali invece, grazie alla maggiore ricchezza di fauna, sia l'agguato che la ricerca attiva possono dare buoni risultati ed infatti, accanto a ragni che fanno tele, incontriamo anche numerose specie che impiegano questi due altri sistemi di caccia.

Sarà opportuno precisare che per "grotta tropicale" io intendo una grotta ricchissima di risorse trofiche, fatto che per lo più si riscontra in grotte situate anche geograficamente in zone tropicali. Naturalmente, in queste regioni, per lo più a quote elevate, possono anche esistere grotte simili dal punto di vista trofico a quelle delle nostre zone, mentre nei paesi temperati a volte ci si può avvicinare a condizioni simili a quelle solite nei tropici.

Per chiarezza è necessario premettere qualche cenno sui ragni delle grotte temperate. Attualmente si hanno conoscenza abbastanza buone sull'Europa (con tutto il Mediterraneo), leggermente minori sulla regione Neartica e sull'Estremo

* Istitu to di Zoologia clell'Università di Roma. 
Oriente (limitatamente alla Corea ed al Giappone); resta la grande lacuna dell'Asia centrale e settentrionale (e minori lacune nel Medio Oriente, specie nell'Iran e nell'Ovest degli Stati Uniti).

La fauna araneologica delle grotte di queste regioni è quanto mai uniforme. Cominciando dai gruppi più primitivi, si possono ricordare i Leptonetidae, in grande maggioranza cavernicoli, assenti dalle regioni tropicali (v.cartina 1); i Dysderidae, meno frequenti in grotta, ma qui rappresentati da non poche forme assai specializzate, limitate alla regione mediterranea (Stalita, Stalagtia, Stalitella ecc.); i Pholcidae, frequentissimi ai tropici, sono presenti in grotta con ben poche specie (Hoplopholcus ecc.). Passando ai ragni filogeneticamente più evoluti, quasi tutti quelli delle grotte temperate appartengono alla grande superfamiglia Araneoidea e più precisamente alle famiglie Araneidae (con le comunissime Meta), Linyphiidae (Leptyphantes, Troglohyphantes, Porrhomma, Centromerus ecc.). Micryphantidae (Diplocephalus ecc.), Theridiidae (Robertus) e Nesticidae (Nesticus, Gaucelmus); il resto è costituito da Agelenidae (Tegenaria, Cicurina ecc.) ed Amaurobiidae(Amaurobius ecc.). Non mancano altre famiglie, ma la grande maggioranza è compresa nei gruppi elencati. Queste specie sono senza dubbio penetrate nelle grotte in vari periodi e si potrebbero (almeno quelle mediterranee, $\mathrm{cfr}$. Brignoli, 1973b) riunire in tre principali gruppi: forme termofile ed igrofile, di clima temperato caldo, più umido di quello mediterraneo attuale (Leptonetidae, forse però non tutti), forme di clima freddo ed umido, da periodo interglaciale freddo, per intenderci (molti Linyphiidae), forme di clima intermedio tra i precedenti, temperato ed umido, "da faggeta" (la maggior parte).

Sarebbe auspicabile poter esaminare anche la fauna delle grotte di clima temperato dell'emisfero Sud (Argentina e Cile meridionali, Australia del SE, Nuova Zelanda ecc.), i dati esistenti però sono ancora talmente scarsi da non permettere alcuna conclusione.
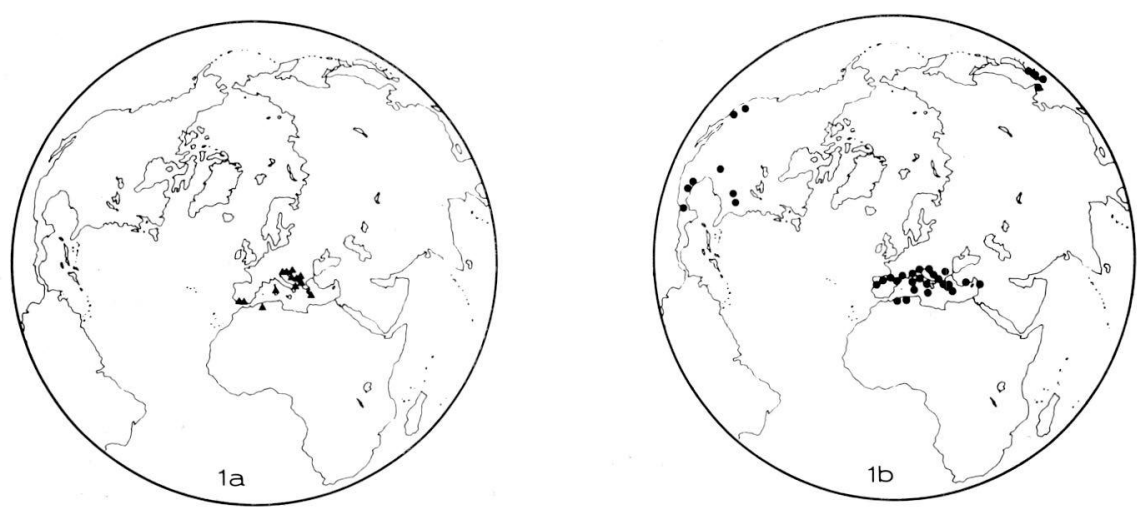

Fig. 1. Due gruppi tipici delle grotte temperate; la: Dysderidae cavernicoli lato sensu; 1b: Leptonetidae, cavernicoli ed epigei. 
Le conoscenze sui ragni delle grotte tropicali non sono molto estese e derivano da raccolte per lo più sporadiche. E' sorprendente quindi che da questi dati si possa già desumere qualcosa e cioè che la fauna delle grotte tropicali è anch'essa notevolmente uniforme, anche se di composizione assai differente.

Si può ricordare, per inciso, che i ragni aumentano notevolmente di numero, come specie, verso i tropici; ben poche sono però le famiglie limitate o ai tropici o alle regioni temperate.

Le regioni sulle quali si ha qualche notizia sono le seguenti: in Asia, l'India settentrionale (zona di confine con la regione paleartica), Ceylon, la Birmania, la Malesia e le Filippine (oltre a sporadici dati su Vietnam, Sumatra ed Okinawa anch'essa zona intermedia-); in Africa, la Guinea, il Congo (sia ex-belga che ex-francese), il Kenya, il Tanganica, Zanzibar (pochi dati su Gabon e Madagascar) nonchè il Sudafrica (che climaticamente è in parte temperato); in America, a parte il Messico, zona di confine sulla quale si sa ormai parecchio, si hanno pochi dati su Cuba, Portorico, Trinidad, Venezuela e Brasile.

Cominciando anche qui dalle forme più primitive, troviamo anzitutto che in queste grotte vivono assai frequentemente degli Ortognati, sia Lifistiomorfi che Migalomorfi. Nel complesso gli Ortognati sono i più simili ai ragni del Paleozoico e si sono separati assai precocemente dalla linea filetica da cui sono derivati gli altri ragni giungendo non di rado ad un notevole grado di specializzazione. Definirli "fossili viventi" non è assolutamente il caso; si tratta di un gruppo che a molti caratteri plesiomorfi ne unisce quasi altrettanti di apomorfi. Il loro indubbio successo evolutivo è attestato dalla loro frequenza in tutto il mondo, salvo che nelle zone più fredde. Possiamo tra essi ricordare Liphistius batuensis Abraham, comunissimo nelle Batu Caves in Malesia nonchè le numerose specie cieche o microftalme finora descritte come, tra i Dipluridae, Accola caeca Simon delle Filippine, Euagrus cavernicola Gertsch del Messico e Troglodiplura lowryi Main dell'Australia del SW, tra i Barychelidae, Troglothele coeca Fage di Cuba, tra i Theraphosidae, Aphonopelma stygia Gertsch del Messico e, tra gli Ctenizidae, Aporoptychus stercoricola Denis (raccolto nel guano) della Guinea. Numerose altre specie, non adattate, sono state trovate in grotte tropicali; già Simon (1893) faceva rilevare la grande abbondanza di Orphnaecus pellitus Simon nella grotta di Calapnitan nelle Filippine; gli amici Argano e Sbordoni, durante le loro ricerche in Messico, hanno spesso avuto la ventura di imbattersi in "migale" di varie specie nelle grotte.

Nei paesi temperati gli Ortognati sono tutt'altro che rari, ma pressochè assenti nelle grotte (tre soli reperti nel Mediterraneo, per quanto mi consta, tutti di specie chiaramente troglossene).

Questi ragni, di solito di dimensioni medie o grandi, sono di rado predatori vaganti; per lo più praticano la caccia all'agguato, stando presso l'imboccatura delle loro tane o in piccole tele ad imbuto di limitata complessità ed efficienza.

Un altro gruppo estremamente frequente nelle grotte tropicali è quello delle cosiddette Haplogynae, gruppo forse polifiletico che ruinisce tutta una serie di famiglie di ragni primitivi sotto vari aspetti (genitali, struttura delle tele ecc.). Si tratta di ragni dai tegumenti spesso sottili ed assai delicati o anche, in molte forme 
detriticole, protetti da scudetti sclerificati (protezione contro la disidratazione). Senza entrare in merito sulla omogeneità di questo gruppo, si può però rilevare che la grande maggioranza delle famiglie e dei generi ad esso attribuiti è tutt'altro che frequente. Moltissime specie sono detriticole; rare o rarissime nel terreno delle regioni temperate (specie dell'emisfero Nord) sono assai più frequenti nelle foreste tropicali, ma per il momento dobbiamo solo accontentarci di prospettarla, giacchè far pensare alla possibilità che si tratti di gruppi in competizione in molti casi con i ragni più evoluti, più resistenti alla disidratazione e le cui tele (o in generale le cui tecniche di caccia) sono più efficienti. Questo ipotesi è di grande interesse, anche per le connessioni che essa ha colle origini del popolamento in ragni delle grotte tropicali, ma per il momento dobbiamo solo accontentarci di prospettarla, giacchè non sappiamo ancora assolutamente nulla sul reale rapporto numerico esistente tra Haplogynae ed Entelegynae (i ragni più evoluti) nel detrito delle foreste tropicali. Le ricerche del de Barros Machado nell'Angola hanno rivelato la frequenza di certi gruppi ritenuti rari di Haplogynae (Telemidae, Ochyroceratidae, Tetrablemmidae), ma hanno anche rivelato l'esistenza, fino ad allora quasi insospettata, di numerosi Micryphantidae assai evoluti nelle stesse zone. Se questi gruppi siano tra loro in competizione o no è ancora del tutto ignoto. Quello che è certo è che quasi ogni ricerca in grotte tropicali ha portato alla scoperta di Haplogynae in esse. Tra tutti i più abbondanti sono gli Oonopidae, mai cavernicoli in regioni temperate (salvo che in Giappone); ricordo le specie cieche Dysderoides typhlos Fage (India settentrionale), Blanioonops patellaris Simon \& Fage (Tanganica), Wanops coecus Chamberlin \& Ivie (Yucatan), nonchè i numerosi reperti di forme non specializzate in grotte di India, Ceylon, Congo, Gabon, Kenya, Zanzibar e Messico. I Tetrablemmidae, anch'essi, come gli Oonopidae, ai quali superficialmente somigliano, di norma

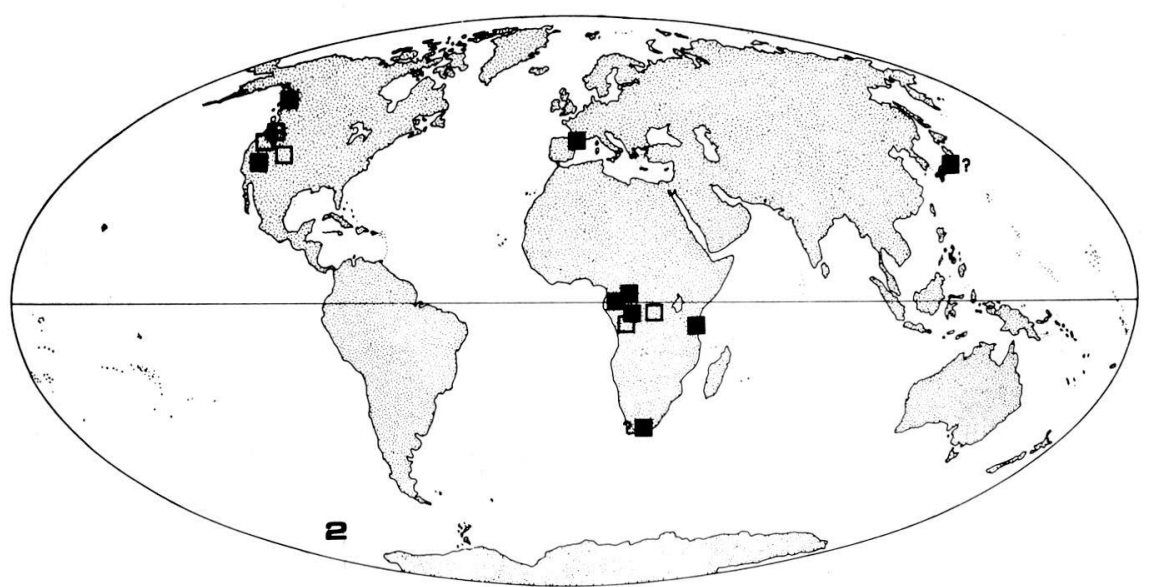

Fig. 2. Distribuzione dei Telemidae (quadrati neri: specie cavernicole, quadrati bianchi: specie epigee); col punto interrogativo i reperti di Cangoderces lewisi Harington e di Merizocera nipponica Yaginuma. 


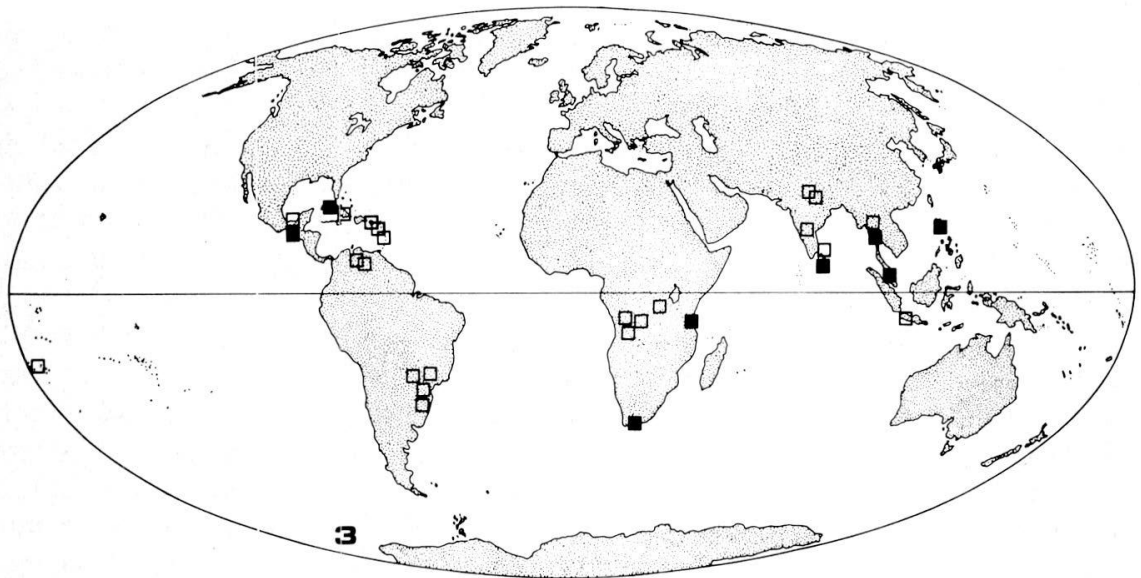

Fig. 3. Distribuzione degli Ochyroceratidae (quadrati neri: specie cavernicole, quadrati bianchi: specie epigee); incluse le due specie del genere Calheirosia Mello Leitao, escluse tutte le specie di Usofïla Keyserling in Marx nonchè Cangoderces lewisi Harington e Merizocera nipponica Yaginuma.

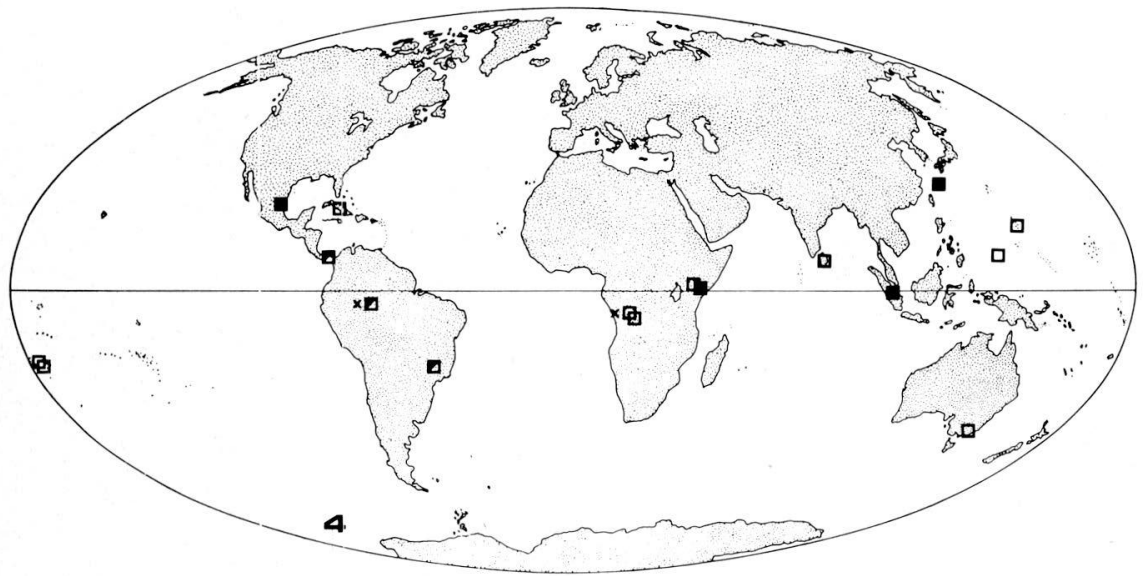

Fig. 4. Distribuzione dei Tetrablemmidae (quadrati neri: specie cavernicole, quadrati bianchi: specie epigee, quadrati metà neri e metà bianchi: specie endogee cieche o con 1-2 occhi soltanto; con una " $\mathrm{x}$ " sono indicati $\mathrm{i}$ dati ancora inediti). N.B. :non sono indicati $\mathrm{i}$ reperti dei Pacullidae sensu Brignoli, 1972a. 
detriticoli, non mancano nelle grotte; ricordo cosi Tetrablemma shimojanai Komatsu (Okinawa) e le specie cieche Tetrablemma sbordonii Brignoli (Messico) ed Ablemma baso Roewer (Sumatra).

Questa famiglia è assai notevole perchè in essa si possono riscontrare tutti i gradi di riduzione oculare nelle forme detriticole: da 6 occhi (condizione normale Hexablemma) si va a forme con 4 (vari Tetrablemma) a 2 (Matta), 1 (Monoblemma) o nessun occhio (una nuova specie dell'Amazzonia raccolta dal Dr.L.Beck/Bochum che ho in studio). Anche negli Oonopidae sono noti fenomeni simili; Berland (1914) ha anzi attribuito alla stessa specie (Triaeris macrophthalmus Berland), detriticola del Kenya, degli individui con occhi progressivamente ridotti, provenienti in parte dalle stesse località, descrivendoli come "forma" media (occhi più piccoli e distanziati) e "forma" cryptops (occhi ridotti, puntiformi). Si possono ricordare poi le specie con 2 soli occhi come Diblemma donisthorpei O.Pickard Cambridge (cfr. Bristowe, 1948) e Dysderina caeca Biraben dell'Argentina, nonchè le non poche cieche termitofile descritte da Benoit (1964) del Congo. In questi ed analoghi casi in cui coesistono nello stesso gruppo forme normalmente oculate e forme con occhi ridotti (cfr. anche vari Agelenidae, come le Cicurina, v. Brignoli, 1972a o i Dictynidae come Scotolathys ecc.) è necessaria estrema prudenza prima di interpretare questo fatto come prova di adattamento alla vita cavernicola; si tratta più probabilmente di forme detriticole e non cavernicole.

Per gli altri gruppi di Haplogynae il problema è leggermente diverso; mentre infatti Oonopidae e Tetrablemmidae sono forme spiccatamente adattate alla vita nel detrito, di modeste o minime dimensioni, dalle zampe brevi, dai tegumenti spesso ricoperti da scudi lisci (protezione evidentemente anche meccanica, oltre che diretta ad evitare la disidratazione), predatori vaganti privi di tela, per quanto ci è noto

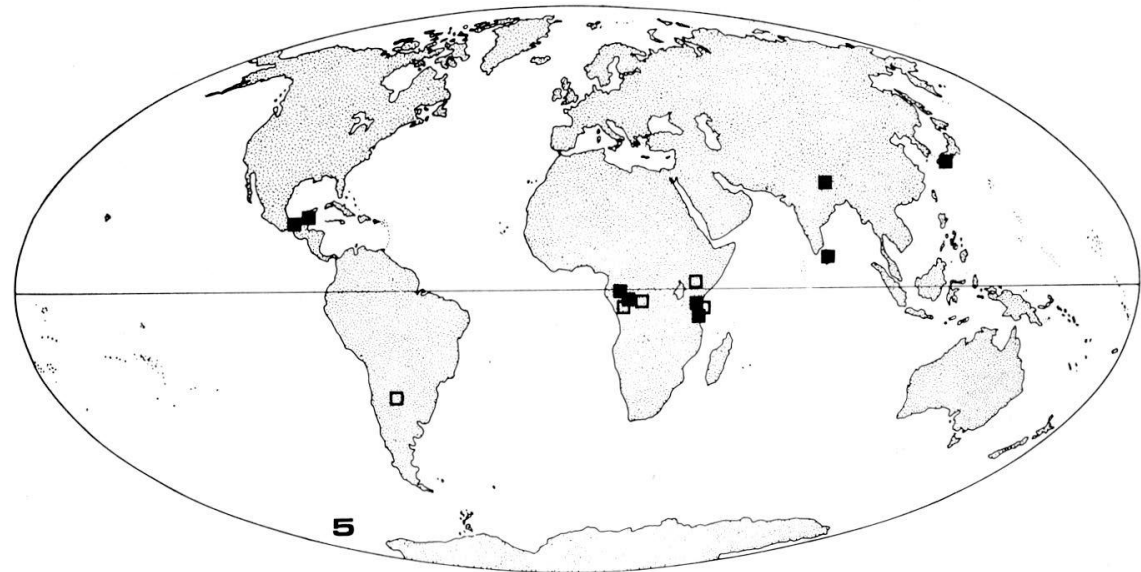

Fig. 5. Gli Oonopidae cavernicoli od endogei o termitofili specializzati (quadrati neri: specie cavernicole, quadrati bianchi: specie endogee o termitofile cieche o con riduzioni oculari). 
(molti, a giudicare anche dai grandi occhi spesso presenti, probabilmente cacciano a vista), altri gruppi, come molti Ochyroceratidae, Telemidae o Pholcidae sono anch'essi detriticoli (e cavernicoli), ma hanno dimensioni per lo più leggermente maggiori, zampe lunghe, tegumenti assai sottili e privi di protezioni e, per quanto è noto, fanno piccole tele. Non credo sia azzardato interpretare questi ragni come dei "microcavernicoli", cioè abitatori non tanto del detrito superficiale, spesso smosso e rimescolato dall'azione degli agenti atmosferici o da altri animali, quanto abitatori delle fessure più o meno permanenti del suolo, delle rocce o anche, per es. dei tronchi morti. Questa ipotesi è suffragata, sia pure in modo assai superficiale, dal fatto che nei molti campioni di fauna del suolo di zone tropicali, ottenuta col vaglio o col selettore di Berlese o analoghi sistemi, che ho avuto modo di esaminare negli ultimi tempi, ben di rado si trovano associati ragni di questi due gruppi; per di più (anche se non è certo una gran prova) mi sembra non privo di significato il fatto che nel materiale di questi campioni da me esaminati (di Amazzonia, Nepal, Ceylon ecc.) Tetrablemmidae ed Oonopidae erano sempre in ottime condizioni, mentre gli Ochyroceratidae od i Pholcidae erano per lo più mutilati. Ciò farebbe supporre appunto che in natura Ochyroceratidae e Pholcidae vivano in ambienti "più tranquilli" di quelli abitati da Tetrablemmidae ed Oonopidae.

Gli Ochyroceratidae sono una famiglia assente dalle zone temperate (Merizocera nipponica, descritta da pochissimo del Giappone da Yaginuma, 1972, per me è probabilmente un Telemida) non rara in grotte tropicali, varie specie (nessuna cieca) sono note di grotte di Filippine, Malesia, Birmania, Ceylon, Tanganica, Sudafrica, Messico e Cuba.

I Telemidae sono invece uno dei gruppi a distribuzione più singolare; ad essi infatti appartengono sia specie cavernicole di zone temperate, come la cieca Telema tenella Simon dei Pirenei o le Usofila degli Stati Uniti (genere da me recentemente spostato dagli Ochyroceratidae a questa famiglia, v.: Brignoli, 1973a, sia cavernicole tropicali (Apneumonella oculata Fage del Tanganica e altre specie ancora inedite di Gabon e Congo, cfr. Lawrence, 1958), sia infine detriticole (lato sensu) tropicali (tutte ancora inedite, cfr. de Barros Machado, 1956).

I Pholcidae, i più evoluti tra le Haplogynae, quanto a struttura della tela e degli organi genitali, sono presenti nelle grotte temperate con poche specie (tra cui per es. il banalissimo Pholcus phalangioides (Fuesslin)) in quelle tropicali invece sono frequentissimi; un certo numero è anche cieco (v.per es. le cinque specie cieche descritte da Gertsich, 1971, del Messico). Definire di origine "detriticola" queste specie cieche è arduo, date le loro dimensioni; può darsi che siano dei "microcavernicoli", ma nemmeno questo è sicuro.

Gli Scytodidae, l'ultimo gruppo di Haplogynae che qui esaminerò, pongono dei problemi ancora diversi. Pochi Scytodes e Loxosceles sono noti di grotte mediterranee, mentre assai numerosi sono i reperti di specie di ambedue i generi (ma più del secondo) in grotte di varie zone tropicali (Malesia, Congo, Sudafrica, Messico, Venezuela e Brasile). Si tratta di ragni di dimensioni medie, mai detriticoli, a volte predatori vaganti o utilizzanti tele poco complesse. Nessuna specie presenta adattamenti di sorta; si tratta quindi di troglofili. Poichè non è nemmeno possibile definirli igrofili è probabile che essi siano un gruppo semplicemente "attirato" nelle 
grotte tropicali dall'abbondanza di possibili prede.

Contro questa ricchissima rappresentanza di ragni "primitivi", sta lo scarsissimo numero di Araneoidea. Finora, a parte i troglosseni, le uniche famiglie presenti con una relativa frequenza nelle grotte tropicali sono $\mathrm{i}$ Theridiosomatidae ed $\mathrm{i}$ Symphytognathidae; assai più rari sono i Nesticidae. I primi, rari nelle regioni temperate e qui mai cavernicoli, sono noti di grotte di Messico, Venezuela, Brasile, Ceylon ed India (Wendilgarda, Andasta), interessante è che alcune specie epigee sono spiccatamente igrofile e vivono tra la vegetazione delle sponde di stagni o corsi d'acqua; i secondi, presenti con poche specie nelle zone temperate (di cui qualcuna cavernicola: Pseudanapis, Trogloneta e Conoculus) non sono rari nelle grotte degli Stati Uniti e del Messico (Maymena); si tratta di un gruppo con tutta probabilità eterogeneo la maggioranza delle cui specie è frequente nei muschi e nel detrito di zone tropicali o subtropicali. I Nesticidae, noti di cavità di Messico, Cuba e Ceylon, sono assai più rari che nelle grotte temperate. Tutte le specie cavernicole di queste famiglie sono di modeste dimensioni, hanno a volte tegumenti parzialmente coperti da scudi e fanno piccole tele.

I Linyphiidae e Micryphantidae, cosî comuni nelle grotte temperate, pur non mancando ai tropici, non sono stati quasi mai raccolti in grotte di queste zone (salvo qualche cavità di quote elevate nell'Africa orientale).

Anche gli Agelenidae ed Amaurobiidae sono praticamente assenti nelle grotte tropicali; fanno eccezione il Sudafrica ed il Messico - regioni del resto climaticamente intermedie - dove non sono rare in grotte rispettivamente delle Phanotea e delle Tegenaria.

Nelle grotte tropicali troviamo invece assai spesso degli Ctenidae, grossi predatori vaganti, dei quali è stata anche descritta una specie microftalma (Trogloctenus fagei de Lessert del Congo), mentre numerose altre sono state citate di cavità del Congo, del Gabon, della Guinea, del Messico e del Venezuela.

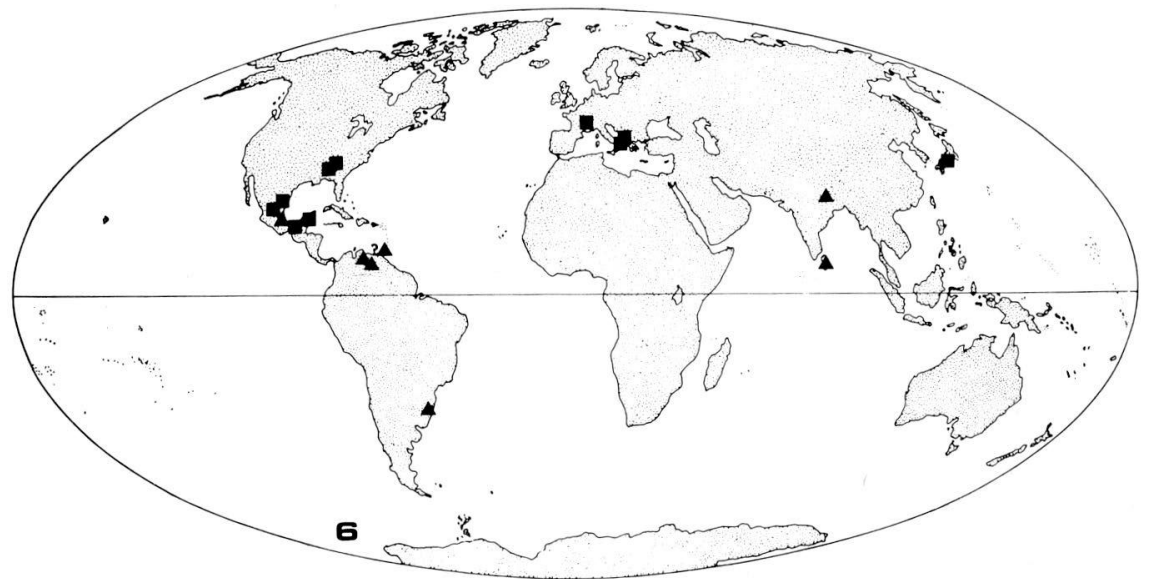

Fig. 6. I Theridiosomatidae (triangoli) ed i Symphytognathidae (quadrati) cavernicoli lato sensu; col punto interrogativo il reperto di Maymena bruneti Gertsch. 
E ancora, tra i cosiddetti Cribellati, mentre nelle grotte temperate solo occasionalmente si possono ancora trovare Zoropsidae, Filistatidae ed Uloboridae, ai tropici non sono rari in grotta sia i Filistatidae (Filippine, Madagascar), sia gli Uloboridae (Malesia, Ceylon, Zanzibar, Venezuela), sia i Dictynidae (Messico, Sudafrica). Tutte queste specie fanno tele notevolmente evolute ed efficienti.

$\mathrm{Da}$ questo rapido esame, nel quale ho trascurato qualche gruppo meno significativo (Clubionidae, Hypochilidae ecc.), appare evidente che la fauna di ragni delle grotte tropicali è assai più variata di quella delle grotte temperate. Dato quanto già detto, che cioè alle nostre latitudini, anche se il numero di specie è inferiore a quello delle zone tropicali, il numero delle famiglie è invece più o meno lo stesso, è chiaro che questa maggiore varietà deve essere dovuta al maggior numero di nicchie presenti in una grotta tropicale. Mi sembra anche che sia evidente una notevole uniformità nel popolamento delle grotte tropicali: come i gruppi presenti in una grotta del Kentucky sono più o meno gli stessi presenti in grotte dell'Italia o del Giappone, cosî i gruppi ritrovati in una grotta del Venezuela corrispondono all'incirca a quelli delle grotte del Tanganica o della Malesia.

Come in quelle temperate, cosî in quelle tropicali mancano finora i rappresentanti di un certo numero di famiglie, per es., limitandoci alle più importanti, i Lycosidae e i Salticidae (tipici cacciatori a vista) e i Thomisidae (cacciatori all'agguato, spesso su fiori).

Dal lato etologico, un notevolissimo numero di cavernicoli tropicali appartiene a gruppi frequenti nel terreno (detriticoli o "microcavernicoli"); tra i cavernicoli di grotte temperate invece questi gruppi sono decisamente meno rappresentati.

Di converso, ben più abbondanti nelle grotte temperate che in quelle tropicali sono le forme che definirei silvicole, appartenenti a gruppi cioè frequenti a varia altezza su alberi, cespugli o erbe alte.

Dal lato etologico infine, nelle grotte tropicali sono piuttosto frequenti i predatori vaganti e quelli utilizzanti l'agguato; relativamente rare invece sembrano essere le forme analoghe alle nostre Meta 'e Tegenaria, di grosse dimensioni cioè e utilizzanti tele ampie e robuste, anche se, in limitata misura gli Uloborus potrebbero essere considerati equivalenti ad esse.

\section{RIASSUNTO}

Le grotte di tipo "tropicale" (per lo più situate anche geograficamente in zone tropicali) si distinguono da quelle di tipo "temperato" essenzialmente per il grande, spesso enorme, numero di risorse trofiche in esse esistenti. I ragni, frequentissimi in ambedue i tipi di grotte, non sono però rappresentati dalle stesse famiglie o dagli stessi generi in ambedue i tipi (benchè la maggior parte delle famiglie abbia una distribuzione estesa sempre ad almeno un'area temperata ed una tropicale). Come in tutte le grotte temperate sono presenti più o meno gli stessi gruppi, cosî le grotte tropicali di tutto il mondo hanno una fauna in ragni piuttosto uniforme, ma costituita da diversi e più numerosi gruppi.

Nelle grotte temperate i gruppi principali sono i Leptonetidae, i Dysderidae, 
moltissimi Araneoidea e molti Agelenidae; questi gruppi sono assenti o scarsamente rappresentati nelle grotte tropicali. In queste ultime invece sono largamente rappresentati gli Ortognati e i ragni primitivi del gruppo delle Haplogynae (Oonopidae, Tetrablemmidae, Ochyroceratidae, Scy todidae, Pholcidae, Telemidae) assieme a pochi Araneoidea (Theridiosomatidae e Symphytognathidae).

Mentre nelle grotte temperate, dal lato ecologico, sono scarsamente numerosi i gruppi tipici del detrito, questi sono invece frequentissimi nelle grotte tropicali; in queste ultime inoltre, accanto a forme letteralmente detriticole, ve ne sono molte definibili più propriamente "microcavernicole" (viventi cioè in piccole cavità più o meno permanenti del suolo). Più frequenti nelle grotte temperate sono invece le forme appartenenti a gruppi viventi tipicamente su piante, a una certa altezza dal suolo. Dal lato etologico, nelle grotte tropicali è possibile l'esistenza di un gran numero di ragni impieganti come metodo di caccia l'agguato o la ricerca attiva (in quelle temperate predominano invece i ragni che fanno tele).

\section{SUMMARY}

The so called "tropical" caves (most of which are also geographically "tropical") are distinguished from the "temperate" caves by the much larger trophic resources. Spiders are common in both kinds of caves, but the groups present in one kind are mostly absent in the other (notwithstanding that most families are distributed over at least one temperate and one tropical region). As in all temperate caves can be found more or less the same groups of spiders, so the tropical caves have a typical spider fauna, composed of different groups (often also more than those present in the temperate caves).

In the temperate caves the most typical groups are the Leptonetidae, the Dysderidae, many Araneoidea and some Agelenidae; these groups are either absent or rare in the tropical caves. In these the typical groups are some Orthognatha and many primitive spiders of the Haplogynae (Oonopidae, Tetrablemmidae, Ochyroceratidae, Scytodidae, Pholcidae, Telemidae) with a few Araneoidea (Theridiosomatidae and Symphytognathidae). From an ecological point of view, the detriticolous groups are not common in temperate caves, but are exceedingly common in tropical caves. In these live also often some groups which could be considered not strictly detriticolous, but more exactly "microcavernicolous" (i.e. living "normally" in more or less permanent crevices etc. of soil and rocks). In temperate caves are on the other hand more common groups living typically on vegetation, not very close to the soil.

Ethologically, in tropical caves the existence of groups is possible which either ambush their prey or search for it actively whereas most spiders of temperate caves capture it with a web. 


\section{BIBLIOGRAFIA}

ABRAHAM, H.C. 1923: A new spider of the genus Liphistius. J. Malay., Br. Asiat. Soc., 1, pp. 13-21.

BARROS MACHADO, A. de, 1956: Captures d'araignées Telemidae au Congo Belge et paleogeographie de cette famille. Folia Scient. Afr. Centr. 2(4), pp. 26-27.

BENOIT, P.L.G. 1964: La découverte d'Oonopides anophthalmes dans les termitières africaines. Rev. Zool. Bot. Afr. 70, pp. 174-187.

BERLAND, L. 1914: Arachnida III, Araneae (lre partie) in "Voy. Alluaud et Jeannel en Afrique Orientale, Res. Scient.", pp. 39-94.

BIRABEN, M. 1954: Nuevas Gamasomorphinae de la Argentina. Notas Mus. Eva Peron, 17, pp. 181-212.

BRIGNOLI, P.M. 19\%2a: Some cavernicolous spiders from Mexico - Quad. Acc. Naz. Lincei, 171, pp. 129-155.

BRIGNOLI, P.M. 1972b: Sur quelques araignées cavernicoles d'Argentine, Uruguay, Brésil et Venezuela récoltées par le Dr P.Strinati. Rev. Suisse Zool., 79, pp. 361-385.

BRIGNOLI, P.M. 1972c: Ragni di Ceylon I. Missione biospeleologica Aellen-Strinati (1970). Rev. Suisse Zool., 79, pp. 907-929.

BRIGNOLI, P.M. 1973a: I Telemidae, una famiglia di ragni nuova per il continente americano. Fragm. Ent., 8, pp. 247-263.

BRIGNOLI, P.M. 1973b: Considerazioni biogeografiche sui ragni cavernicoli mediterranei. C.R.5e Congr. Intern. Arachn. Brno, (1971), pp. 79-84.

BRIGNOLI, P.M. 1973 ? : Note su ragni, principalmente cavernicoli, del Messico meridionale e del Guatemala. Quad. Acc. Naz. Lincei, (in stampa).

BRISTOWE, W.S. 1948: Notes on the structure and systematic position of Oonopid spiders based on examination of the British species. Proc. Zool. Soc. London, 118, pp. 878-891.

BRISTOWE, W.S. 1952: The Arachnid fauna of the Batu Caves in Malaya. Ann. Mag. Nat. Hist., (12) 5, pp. 697-707.

CHAMBERLIN R.V. and W. IVIE, 1938: Araneida from Yucatan. Publ. Carnegie Inst. Wash., 491, pp. $123-136$.

DENIS, J. 1955: Quelques araignées cavernicoles de Guinée francaise. Bull. I.F.A.N., 17, pp. 1024-1033.

FAGE, L. 1912: Etudes sur les araignées cavernicoles. I. Revision des Ochyroceratidae (n. fam.). Arch. Zool. Expér. Génér., (5) 10 (Biospeologica 25), pp. 97-162.

FAGE, L. 1924: Aranıids from the Siju Cave, Garo Hills, Assam. Rec. Ind. Mus., 26, pp. 63-67.

FAGE, L. 1929: Fauna of the Batu Caves, Selangor, X. Arachnida : Pedipalpi (Part) and Araneae. J. Fed. Malay. St. Mus., 14, pp. 356-364.

FAGE, L. 1929: Sur quelques araignées des grottes de l'Amérique du Nord et de Cuba. Boll. Lab. Zool. Portici, 22, pp. 181-187.

FAGE, L. 1931: Araneae, 5e série, précédée d'un essai sur l'évolution souterraine et son déterminisme. Arch. Zool. Expér. Génér., 71 (Biospeologica 55), pp. 91-291.

FAGE, L. 1945: Arachnides cavernicoles nouveaux du Madagascar. Bull. Mus. Nat. Hist. Nat. Paris, 17 , pp. 301-307.

FAGE, L. 1946: Araignées cavernicoles de 1'Inde. Bull. Mus. Nat. Hist. Nat. Paris, 18, pp. 382-388.

GERTSCH, W.J. 1960: Description of American spiders of the family Symphytognathidae. Amer. Mus. Novit., 1981, pp. 1-40.

GERTSCH, W.J. 1971: A report on some Mexican cave spiders. Bull. Ass. Mex. Cave. St., 4, pp. 47-111.

HARINGTON, J.S. 1951: A new Leptonetid spider, Cangoderces lewisi n. gen. n. sp. from the Cango Caves, Oudtshoorn. Ann. Natal Mus., 12, pp. 81-90.

KOMATSU, T. 1968: Two new spiders of genera Tetrablemma and Dolichocybaeus. Acta Arachnol. Osaka, 21(2), pp. 35-39. 
LAWRENCE, R.F. 1951: The cave-living spiders of the South African genus Phanotea Simon. Rev. Zool. Bot. Afr., 45, pp. 49-54.

LAWRENCE, R.F. 1952: A collection of cavernicolous and termitophilous Arachnida from the Belgian Congo. Rev. Zool. Bot. Afr., 46, pp. 1-17.

LAWRENCE, R.F. 1958: A collection of cavernicolous Arachnida from French Equatorial Africa. Rev. Suisse Zool., 65, pp. 857-866.

LAWRENCE, R.F. 1964: New cavernicolous spiders of South Africa. Ann. South Afr. Mus., 48, pp. 57-75.

LAWRENCE, R.F. 1967: A new cavernicolous Pholcid spider from the Congo. Rev. Suisse Zool., 74, pp. 295-300.

LESSERT, R. de, 1935: Description de deux araignées cavernicoles du Congo Belge. Rev. Zool. Bot. Afr., 27, pp. 326-332.

MAIN, B.Y. 1969: A blind Mygalomorph spider from a Nullarbor Plain cave. J. R. Soc. West. Austr., 52, pp. 9-11.

ROEWER, C.F. 1962: Einige Arachniden aus den Batu Caves in Malaya. Pacif. Insects 4(2), pp. 517-520.

ROEWER, C.F. 1963: Ueber einige neue Arachniden der orientalischen und australischen Region. Senckenberg. biol., 44, pp. 223-230.

SIMON, E. 1893: Arachnides in "Voy. de M.E.Simon aux iles Philippines". Ann. Soc. Ent. France, 62, pp. 65-80.

SIMON, E. 1894: Note sur les Arthropodes cavernicoles du Transvaal. Ann. Soc. Ent. France, 63, pp. 63-67.

SIMON, E. 1906: Description d'un Arachnide cavernicole du Tonkin. Bull. Soc. Ent. France, 1906(2), p. 27.

SIMON, E. and L. FAGE, 1922: Araneae des grottes de l'Afrique Orientale. Arch. Zool. Expér. Génér., 60 (Biospeologica 44), pp. 523-555.

STRINATI, P. 1971: Recherches biospéologiques en Amérique du Sud. Ann. Spéléol., 26, pp. 439-450.

YAGINUMA, T. 1972: The fauna of the Lava Caves around Mt. Fuji-san. IX. Araneae. Bull. Nat. Sc. Mus. Tokyo, 15, pp. 267-334. 